\title{
MicroRNA-155 and Anti-Müllerian Hormone: New Potential Markers of Subfertility in Men with Chronic Kidney Disease
}

\author{
Dag Eckersten $^{a} \quad$ Christos Tsatsanis $^{b, c}$ Aleksander Giwercman ${ }^{c}$ \\ Laila Bruun $^{a} \quad$ Mats Pihlsgård $^{d}$ Anders Christensson $^{a}$ \\ a Department of Nephrology, Lund University, Skåne University Hospital, Malmö, Sweden; \\ ${ }^{b}$ Department of Clinical Chemistry, School of Medicine, University of Crete, Heraklion, \\ Crete, Greece; ' Molecular Reproductive Medicine, Department of Translational Medicine, \\ Lund University, Malmö, Sweden; ${ }^{\mathrm{d}}$ Department of Geriatrics, Lund University, Skåne \\ University Hospital, Malmö, Sweden
}

Keywords

Hemodialysis · Fertility · Chronic kidney disease

\section{Abstract}

Background/Aims: Men with terminal renal failure are often infertile. Anti-müllerian hormone $(\mathrm{AMH})$, a marker of Sertoli cell function, is decreased among men with chronic kidney disease (CKD). Recently, a microRNA, miR-155, has been shown to be a potential marker for subfertility. We studied miR-155 and semen parameters in patients with CKD who were not yet on dialysis. We also aimed to study possible associations between AMH, miR-155, and semen parameters to evaluate them as markers of fertility. Methods: Thirty male patients with CKD 1-4 as well as 18 healthy controls were enrolled. Results: Serum levels of miR-155 were significantly higher among men with CKD stages $1-2(4.51 \pm 3.81[p=0.01])$ and stages $3-4$ $(2.75 \pm 1.77[p=0.006])$ than in controls $(1.09 \pm 0.44)$. Sperm concentration was significantly lower among men with CKD 3-4 (42 \pm 29$) \times 10^{6} / \mathrm{mL}$ compared to controls $(88 \pm 42) \times 10^{6} / \mathrm{mL}$ $(p=0.011)$. High levels of miR-155 were associated with a relatively low sperm concentration $(p=0.02)$ and with a low total sperm number $(p=0.005)$. Low AMH levels were associated with a decreased percentage of motile sperm cells $(p=0.02)$. Conclusions: We conclude that men with stage 3-4 CKD had lower sperm concentrations than healthy fertile men and that increased serum miR-155 in men with stage 1-4 CKD was associated with semen parameters that indicate subfertility. Low AMH levels were associated with a low percentage of the total number of motile sperm cells. miR-155 and AMH may be potential markers of subfertility in men with CKD. 
Eckersten et al.: MicroRNA-155 and Anti-Müllerian Hormone: New Potential Markers of Subfertility in Men with Chronic Kidney Disease

\section{Introduction}

Infertility of women with end stage renal disease (ESRD) has been extensively investigated, but reproductive function in men with ESRD is less well characterized, and few data are available in either men or women with earlier stages of chronic kidney disease (CKD). CKD is divided into 5 stages. CKD 1 represents kidney damage with preserved kidney function, whereas CKD 5 indicates severely decreased kidney function that commonly requires dialysis. In men with ESRD, semen analysis often reveals a low ejaculate volume, low sperm count or azoospermia, and decreased sperm motility [1,2]. The cause of subfertility or infertility of men with ESRD is multifactorial. Autonomic dysfunction, endocrine disturbances, testicular dysfunction, vascular disease, psychological factors, and side effects of pharmacologic treatment are frequently involved. Deterioration of kidney function may also imply disturbances of the hypothalamic-pituitary-gonadal axis with increased levels of luteinizing hormone and follicle-stimulating hormone and decreased testosterone levels [3-5]. We have previously shown that anti-müllerian hormone (AMH), a marker of Sertoli cell function, is decreased in all men with CKD [6], being most pronounced in CKD 5 [7]. Low AMH levels have also been seen in subfertile men without renal disease [8] and in men with nonobstructive azoospermia [9]. Furthermore, CKD is associated with low-grade systemic inflammation (LGSI) characterized by retention of various cytokines and creation of a proinflammatory milieu [10] that may also influence fertility. MicroRNAs (miRNAs) are small, noncoding RNAs that control mRNA translation and stability. miRNAs play important roles in the regulation of cell activation, proliferation, and apoptosis [11]. In immune cells, miRNAs have been identified as key regulators of inflammation. Two of these, miR-155 and miR-146a, have central roles in innate immunity $[12,13]$. miRNAs can be secreted from cells within exosomes, or as paracrine or endocrine messengers, and they can be detected in serum as circulating miRNAs [14]. Circulating miRNAs have been accepted as biomarkers for a number of diseases and inflammatory conditions such as LGSI. Diagnosis of male subfertility is based primarily on semen testing, which is often performed after a couple has unsuccessfully attempted to have children. Developing a serum test will allow screening of the affected population and timely intervention, and miRNAs in the serum are a family of such biomarkers.

Previous studies have found that male subfertility was associated with low testosterone levels [15] and LGSI [16]. We recently showed that increased miR-155, but not miR-146a, was associated with male subfertility independent of LGSI or androgens in men without CKD [17].

We evaluated semen parameters at early stages of CKD and miR-155 as a new potential marker of fertility. We also investigated possible associations between AMH and semen parameters because AMH is substantially decreased in men with CKD. Because little is known of fertility in men with early-stage CKD and it is important to effectively identify early-stage CKD patients who are subfertile, we investigated the value of miR-155 and AMH as predictors of early-stage, CKD-associated male subfertility.

\section{Subjects and Methods}

From June 2009 to May 2012, 30 male patients with CKD stages 1-4, aged between 26 and 47 years with a median age of 37 years, were consecutively enrolled at the Department of Nephrology, Skåne University Hospital, Malmö, Sweden. The patients were asked to leave blood samples, 2 semen samples, and to perform iohexol clearance measurements. All 30 patients donated blood samples and performed iohexol clearance measurements, and 21 donated semen samples. Nine out of 30 patients with CKD 1-4 did not donate semen samples. 
Eckersten et al.: MicroRNA-155 and Anti-Müllerian Hormone: New Potential Markers of Subfertility in Men with Chronic Kidney Disease

Table 1. Patient characteristics and renal function

\begin{tabular}{llll}
\hline & Group 1 (controls) & Group 2 (CKD 1-2) & Group 3 (CKD 3-4) \\
\hline Subjects, $n$ & 18 & 16 & 14 \\
Age & $37(26-44)$ & $35(26-42)$ & $37(29-47)$ \\
Cystatin C, mg/L & $0.67(0.11)$ & $0.98(0.18)$ & $2.03(0.56)$ \\
Iohexol, mL/min/1.73 m ${ }^{2}$ & - & $91(15)$ & $35(15)$ \\
CRP mg/L & - & $0.81(0.84)$ & $2.04(1.49)$ \\
Semen samples, $n$ & 18 & 11 & 10 \\
\hline
\end{tabular}

Age is expressed as median (range). Cystatin C, iohexol clearance, and CRP are expressed as means (standard deviation). CKD, chronic kidney disease; CRP, C-reactive protein.

In order to exclude selection bias, we performed ANOVA analysis and found no differences in the reproductive hormone levels between participants and non-participants.

Determination of CKD stages was based on measurements of iohexol clearance. The patients were divided into 3 groups according to Table 1. The first group was the control group.

In this group, we selected partners to pregnant women who achieved pregnancy after less than 12 months of unprotected intercourse, without use of assisted reproduction. Among these 145 proven fertile men we randomly selected 18 age-matched controls. The median age of the controls was 37 years (26-44 years). All subjects in the control cohort presented with normal cystatin $C$ values (mean: 0.68 [SD 0.11] $\mathrm{mg} / \mathrm{L}$ ) and were thus considered to have normal renal function (Table 1). This control group from the Reproductive Medicine Centre, Skåne University Hospital, Malmö, Sweden, has been described previously [18]. Group 2 was composed of patients with kidney disease and iohexol clearance above $59 \mathrm{~mL} / \mathrm{min} / 1.73 \mathrm{~m}^{2}$ (CKD 1-2). Group 3 was composed of patients with iohexol clearance between 15 and $59 \mathrm{~mL} /$ $\mathrm{min} / 1.73 \mathrm{~m}^{2}$ (CKD 3-4). The patients in the groups 2 and 3 had a normal panorama of various kidney diseases. None of the patients were known to suffer from systemic lupus erythematosus or hepatitis infection. All blood samples were drawn in the morning between 8 and 9 a.m. Laboratory analyses are thoroughly described below.

The study was approved by the regional Ethics Committee at Lund University, Sweden (LU 541/2008). All study participants provided written informed consent. The study was conducted in accordance with the principles of the Declaration of Helsinki.

\section{Statistical Analysis}

Analyses were performed using SPSS version 22.0 (IBM Corp., Armonk, NY, USA) and the SAS statistical software version 9.4 (SAS, Cary, NC, USA). A $p$ value $<0.05$ was considered statistically significant.

A Kruskal Wallis test was performed to evaluate differences between the groups regarding miR-155 since this parameter is not normally distributed. Regarding semen parameters and $\mathrm{AMH}$, we initially performed ANOVA to evaluate if there were any differences among the groups (Table 2). However, ANOVA did not show which groups differed from each other. Because ANOVA showed statistical significance for sperm concentration and total sperm count for each analyte, we performed Tukey post hoc analyses to evaluate which groups differed statistically significantly from each other. In order to assess the relationships between semen parameters and miR-155 and $\mathrm{AMH}$, respectively, we conducted linear regression analyses, adjusted for age, between mir-155 and AMH as independent and semen parameters as dependent parameters, respectively 
Table 2. Results of analyses

\begin{tabular}{lcccl}
\hline & Controls & CKD 1-2 & CKD 3-4 & $p$ value \\
\hline miR-155, AU & $1.09(0.44)$ & $4.51(3.80)$ & $2.75(1.77)$ & $0.01^{\text {a; }} 0.006^{\mathrm{a}}$ \\
Sperm concentration, $10^{6} / \mathrm{mL}$ & $88(42)$ & $54(39)$ & $42(29)$ & 0.008 \\
Total sperm count, $10^{6}$ & $266(109)$ & $189(183)$ & $111(88)$ & 0.016 \\
Semen volume, $\mathrm{mL}$ & $3.3(1.3)$ & $3.0(1.5)$ & $2.4(0.8)$ & 0.25 \\
Percentage motile sperm, \% & $65(16)$ & $55(23)$ & $47(23)$ & 0.068 \\
DFI, \% & $10.6(4.9)$ & $15.9(15.7)$ & $15.0(9.7)$ & 0.37 \\
AMH, $\mu \mathrm{g} / \mathrm{L}$ & $9.5(3.6)$ & $8.3(4.8)$ & $7.5(4.4)$ & 0.49 \\
\hline
\end{tabular}

Values are expressed as means (standard deviation). miR, microRNA; AU, arbitrary units; DFI, DNA fragmentation index; $\mathrm{AMH}$, anti-müllerian hormone. ${ }^{\mathrm{a}}$ Kruskal-Wallis test compares controls with chronic kidney disease (CKD) 1-2 and CKD 3-4. Other variables tested by ANOVA.

\section{miRNA Expression}

Serum from participating individuals was isolated and stored at $-80^{\circ} \mathrm{C}$ immediately, and miRNA detection was performed as described previously [17]. Since no internal reference miRNAs exist in the serum, we exogenously introduced a control miRNA (UniSp2miRNA), according to the manufacturer instructions (Exiqon, Vedbaek, Denmark). RNA was extracted using miRCury ${ }^{\mathrm{TM}}$ RNA isolation columns for biofluids (Exiqon). Contaminating cells or cell debris were/was carefully removed by 2 centrifugations: one at the stage of serum isolation and a second one by centrifugation at $3,000 \mathrm{~g}$ prior to isolation of serum RNA. CDNA was synthesized, and levels of miR-155 were measured by real-time PCR according to the manufacturer's instructions using primers validated by the manufacturer for selective amplification of the target miRNAs (Exiqon). Briefly, cDNA prepared with the miRCURY ${ }^{\mathrm{TM}}$ CDNA synthesis kit II (Exiqon) was diluted 1:20; $4 \mu$ l were added to Exilent SYBRgreen PCR master mix (Exiqon) and subjected to real-time PCR under the following conditions: 1 cycle at $95^{\circ} \mathrm{C}$ for $10 \mathrm{~min}, 45$ cycles of $95^{\circ} \mathrm{C}$ for $10 \mathrm{~s}, 60^{\circ} \mathrm{C}$ for $1 \mathrm{~min}$, followed by a dissociation cycle in a Stratagene Mx3000p instrument. The no-template control did not produce any product. Results were analyzed using the Stratagene MxPro software, and the relative miRNA levels in the serum were measured using the $2^{-(\Delta \Delta \mathrm{Ct})}$ method as previously described [17] using as baseline cycle threshold the mean of the cycle threshold values of the control group.

\section{Cystatin $C$}

Plasma cystatin $\mathrm{C}$ levels were measured by a fully automated particle-enhanced immunoturbidimetric assay. The reagents were obtained from Dako (Dako A/S, Glostrup, Denmark), and determination of cystatin $\mathrm{C}$ levels was performed on the Hitachi Modular P analysis system. The total analytical imprecision was $2.1 \%$ for a control sample at a concentration of $1.0 \mathrm{mg} / \mathrm{L}$ and $1.7 \%$ for a control sample at $4.0 \mathrm{mg} / \mathrm{L}$. The reference range was as follows: $0.55-1.15 \mathrm{mg} / \mathrm{L}$ for the age of $1-50$ years and $0.63-1.44 \mathrm{mg} / \mathrm{L}$ for the age $>50$ years [19].

\section{Anti-Müllerian Hormone}

AMH was analyzed at the Department of Clinical Chemistry at Karolinska University Hospital, Stockholm, Sweden. AMH in serum was determined by ELISA using a commercial kit (Ansh Labs Ultrasensitive AMH/MIS ELISA) with a detection limit of $0.023 \mu \mathrm{g} / \mathrm{L}$ and within- and between-assay coefficients of variation (CVs) of 4 and 5\%, respectively. The assay had a mean linearity of $98 \%$ over the dilution range of 1:2-1:16. 
Eckersten et al.: MicroRNA-155 and Anti-Müllerian Hormone: New Potential Markers of Subfertility in Men with Chronic Kidney Disease

\section{Measured Glomerular Filtration Rate}

Glomerular filtration rate (GFR) was determined by measuring the plasma clearance of iohexol according to a one-compartment model. Samples were taken after the distribution phase in the assumed monoexponential part of the plasma decay curve. Iohexol was analyzed using high-performance liquid chromatography $[20,21]$. The reference range was $80-125$ $\mathrm{mL} / \mathrm{min} / 1.73 \mathrm{~m}^{2}$ for this age of patients.

\section{C-Reactive Protein}

Samples were sent to the laboratory at the Department of Clinical Chemistry at the Skåne University Hospital, Malmö, Sweden, where it was immediately analyzed according to standard procedures. Plasma C-reactive protein (CRP) was measured by rate turbidimetry. The detection limit was $0.2 \mathrm{mg} / \mathrm{L}$, and the interassay CVs were $6 \%$ at $15 \mathrm{mg} / \mathrm{L}$ and $5 \%$ at $85 \mathrm{mg} / \mathrm{L}$.

\section{Semen Analyses}

In the collecting and analyzing of the semen samples, the investigators followed the World Health Organization's guidelines [22], which was the latest version of the manual at the time sample collection was initiated. The laboratory at the Skane University Hospital is one of the reference laboratories in the European Society of Human Reproduction and Embryology/Nordic Association for Andrology external quality control program [23].

Semen volume, sperm concentration, total number of sperm per ejaculate, and sperm motility were determined. Sperm DNA fragmentation index (DFI) was evaluated using the sperm chromatin structure assay (SCSA) [24].

The participants were asked to keep an abstinence period of 2-7 days. Semen samples were obtained at the RMC or at home. In the latter case, specimens were brought to the laboratory within $60 \mathrm{~min}$ from the time of ejaculation. Most of the men with CKD delivered 2 samples. The first sample, on which SCSA was performed, was taken into consideration in the statistical analysis performed in this paper. Only 1 ejaculate was obtained from the fertile men. We analyzed semen volume, sperm concentration, and motility. Following liquefaction, $200 \mu \mathrm{L}$ of the ejaculate were stored at $-80^{\circ} \mathrm{C}$ for subsequent SCSA analysis.

\section{Sperm Chromatin Structure Assay}

The details of these methods have previously been described [24, 25]. Shortly, the SCSA is based on the phenomenon that chromatin with DNA strand breaks has a tendency to denature when exposed to an acid detergent, whereas normal chromatin remains stable. The SCSA measures the denaturation of sperm DNA using the dye acridine orange. Acridine orange is a metachromatic dye, which differentially stains double- and single-stranded nucleic acids. After blue-light excitation, the intact (double-stranded) DNA emits green fluorescence, whereas denatured (single-stranded) DNA emits red fluorescence.

Five thousand cells were analyzed by FACSort (Becton Dickinson, San Jose, CA, USA).

Off-line analysis of the flow cytometric data was carried out by using dedicated software (SCSASoft; SCSA Diagnostics, Brookings, SD, USA). The percentage of sperm with detectable DFI (\%DFI) was calculated from the DFI frequency histogram obtained from the ratio between the red and total (red plus green) fluorescence intensity. DFI is the proportion of cells containing denatured DNA [25]. The intralaboratory CV was found to be $4.5 \%$ for DFI. 


\section{Results}

The patient characteristics are shown in Table 1. The mean ages of the study groups were similar and plasma cystatin C levels increased at each CKD stage, as expected. The miR-155 level was significantly higher in men with CKD $1-2(4.51 \pm 3.81 ; p=0.01)$ and CKD 3-4 (2.75 $\pm 1.77 ; p=0.006)$ than in controls $(1.09 \pm 0.44)$ (Table 2 ). There was no significant difference in the miR-155 levels of CKD 1-2 and CKD 3-4 patients ( $p=0.30)$.

ANOVA showed significant differences in sperm concentration and total sperm count (Table 2). The sperm concentration in CKD 1-2 patients was $(54 \pm 39) \times 10^{6} / \mathrm{mL}$, which was lower than that in controls $(88 \pm 42) \times 10^{6} / \mathrm{mL}$, but the difference was not significant (Tukey post hoc, $p=0.07)$. The sperm concentration in CKD 3-4 patients $(41 \pm 29) \times 10^{6} / \mathrm{mL}$ was significantly lower than that in controls (Tukey post hoc, $p=0.01$ ). The total sperm count was also significantly lower in CKD 3-4 patients than in controls (Tukey post hoc, $p=0.01$ ). The mean DFIs and semen volumes of the study groups were not significantly different. There was a tendency towards a lower percentage of motile sperm in CKD 3-4 patients than in controls, but it did not reach statistical significance (Tukey post hoc, $p=0.06$ ). Regression analysis revealed that miR-155 was negatively associated with sperm concentration $(\beta=$ $-0.017, p=0.02)$ and total sperm count $(\beta=-0.006 ; p=0.005)$. AMH was positively associated with the percentage of motile sperm cells $(\beta=0.063 ; p=0.02)$. We did not find a significant association between miR-155 and AMH, miR-155 and CRP, or CRP and AMH. We also did not find significant associations between DFI and CRP, DFI and miR-155, or DFI and $\mathrm{AMH}$.

\section{Discussion}

We found a significantly lower sperm concentration and total sperm count in men with CKD 3-4 than in healthy fertile controls. There was a tendency towards a lower sperm concentration in men at earlier stages (CKD 1-2), but the difference was not statistically significant. These novel data show that a moderate reduction in kidney function can influence male sperm parameters. We also showed that men with CKD demonstrated increased serum miR-155.

miR-155 was recently shown to be a potential marker for subfertility in patients without CKD. miR-155 is a miRNA associated with inflammation $[12,13]$, which is prominent in CKD patients. Nevertheless, Tsatsanis et al. [17] reported that elevated miR-155 levels were associated with male subfertility independent of inflammation and testosterone levels. We found that miR-155 was significantly higher in men with CKD 1-2 and CKD 3-4 than in controls, and that high miR-155 levels were negatively associated with sperm concentration and total sperm count. We found no association between miR-155 and CRP in this CKD population, indicating that the elevated miR-155 levels were a response to a mechanism other than inflammation associated with CRP as a cause of impaired semen quality. We have recently reported major decreases in plasma levels of $\mathrm{AMH}$, a marker of Sertoli cell function, in men with CKD $[6,7]$. To evaluate this in relation to fertility, we included assay of AMH levels in this study, and found an association between low AMH levels and a low percentage of motile sperm cells in the entire study population. We hypothesize that Sertoli cell function was impaired by CKD. Because Sertoli cells support and nourish spermatogenesis, it is plausible that a disturbance in Sertoli cell function could influence sperm motility. The finding of an association between AMH level and sperm motility suggests that AMH might be a subfertility marker in men with CKD. Goulis et al. [8] have previously reported low AMH levels in other groups of subfertile men. To date, the role of AMH in adult men is unknown. Previous studies, 
Eckersten et al.: MicroRNA-155 and Anti-Müllerian Hormone: New Potential Markers of Subfertility in Men with Chronic Kidney Disease

including ours, have only focused on associations and not identified any causative relations between male fertility and AMH.

We did not find an association between AMH and miR-155, suggesting that these are independent markers of subfertility. MiR-155 was associated with the concentration of spermatozoa, and AMH was associated with sperm motility. Low AMH and elevated miR-155 levels in CKD patients may reflect different disturbances affecting the sperm; both may be found useful in the future to identify subfertile patients with early-stage CKD. Altered Sertoli cell function is probably not the only reason for subfertility in men with CKD.

High oxidative stress is an essential mechanism underlying male infertility [26]. Oxidative stress reflects an imbalance between the antioxidant activity and the generation of oxidants in favor of the later [27]. Oxidative stress damages sperm DNA, resulting in a high DFI. In patients with CKD, disturbance in the balance between pro- and antioxidant capacities results in increased oxidative stress [28]. However, in this study DFI was not significantly higher in men with CKD than in controls (Table 2). Nevertheless, we cannot exclude the possibility that oxidative stress might contribute to male subfertility in CKD by mechanisms other than those related to sperm DNA integrity. Larger studies are necessary to evaluate possible correlations between DFI, oxidative stress, and subfertility in men with CKD.

Taken together, the study findings provide new insight and knowledge of parameters indicating subfertility in male CKD patients and suggest a multifactorial origin. Previous explanations include disturbances in the hypothalamic-pituitary-gonadal axis resulting in altered levels of luteinizing hormone, follicle-stimulating hormone, and testosterone. Our findings in this and previous studies [6, 7] show that the absolute change in plasma AMH and miR-155 levels were greater than those of testosterone. Successful renal transplantation has been shown to normalize testosterone deficiency in uremic men [29]. However even though renal transplantation seems to improve male fertility and semen parameters, there are reports of renal transplanted men with remaining severe deficiency of sperm motility [30]. This is consistent with our finding that a moderate reduction in kidney function influences the Sertoli cell function and sperm motility.

Our study has both limitations and strengths. The small study population limited the power of the statistical analyses, and the cross-sectional study design makes it possible to show associations, but not causal relationships, between parameters. However, the substantial, significant increase of miR-155 minimizes the disadvantage of studying a small number of patients. This study also excluded recently and previously transplanted patients as well as patients receiving immunosuppressive drugs, both of which could affect the reliability of the results.

In conclusion, this study shows for the first time that male fertility might be disturbed during the early stages of CKD and that sperm concentration and total sperm count were already affected in patients with moderate renal failure (CKD 3-4). We also showed that miR-155 was upregulated in men with CKD and that it may reflect subfertility in those patients. Furthermore, AMH, previously shown to be decreased in men with CKD, was positively associated with the percentage of motile sperm cells. This association might indicate that low AMH levels could reflect disturbed Sertoli cell function and that AMH could be a marker of subfertility in men with CKD. These findings warrant further exploration in a larger population to confirm our initial findings. 


\section{Acknowledgments}

This work was supported by grants from the Fulbright Commission, the Faculty of Medicine at Lund University, Swedish Renal Association, Research Funds of Region Skåne, and the Research Fund of Skåne University Hospital.

We would like to thank Gun-Britt Eriksson, Mona Hassan Al-Battat at the Wallenberg Laboratory, and Therese Nilsson and Agneta Emilsson at the Department of Nephrology, all at the Skåne University Hospital, Malmö, Sweden.

\section{Disclosure Statement}

The authors declare no conflicts of interest.

\section{References}

1 Prem AR, Punekar SV, Kalpana M, Kelkar AR, Acharya VN: Male reproductive function in uraemia: efficacy of haemodialysis and renal transplantation. Br J Urol 1996;78:635-638.

$>2$ Holdsworth S, Atkins RC, de Kretser DM: The pituitary-testicularaxis in men with chronic renal failure. N Engl J Med 1977;296:1245-1249.

-3 Lim VS, Fang VS: Restoration of plasma testosterone levels in uremic men with clomiphene citrate. J Clin Endocrinol Metab 1976;43:1370-1377.

-4 Albaaj F, Sivalingham M, Haynes P, McKinnon G, Foley RN, Waldek S, O’Donoghue DJ, Kalra PA: Prevalence of hypogonadism in male patients with renal failure. Postgrad Med J 2006;82:693-696.

-5 Gómez F, de la Cueva R, Wauters JP, Lemarchand-Béraud T: Endocrine abnormalities in patients undergoing long-term hemodialysis. The role of prolactin. Am J Med 1980;68:522-526.

-6 Eckersten D, Giwercman A, Bruun L, Christensson A: Anti-müllerian hormone, a Sertoli cell-derived marker, is decreased in plasma of male patients in all stages of chronic kidney disease. Andrology 2015;3:1160-1164.

7 Eckersten D, Giwercman A \& Christensson A: Male patients with terminal renal failure exhibit low serum levels of anti-müllerian hormone. Asian J Androl 2015;17:149-153.

-8 Goulis DG, Iliadou PK, Tsametis C, Gerou S, Tarlatzis BC, Bontis IN, Papadimas I: Serum anti-müllerian hormone levels differentiate control from subfertile men but not men with different causes of subfertility. Gynecol Endocrinol 2008;24:158-160.

-9 Muttukrishna S, Yussoff H, Naidu M, Barua J, Arambage K, Suharjono H, Sathanandan M: Serum anti-müllerian hormone and inhibin B in disorders of spermatogenesis. Fertil Steril 2007;88:516-518.

10 Carrero J.J, Stenvinkel P: Inflammation in end-stage renal disease - what have we learned in 10 years? Seminars in Dialysis 2010;23:498-509.

11 O’Neill LA, Sheedy FJ, McCoy CE: MicroRNAs: the fine-tuners of Toll-like receptor signalling. Nat Rev Immunol 2012;11:163-175.

12 Arranz A, Doxaki C, Vergadi E, Martinez de la Torre Y, Vaporidi K, Lagoudaki ED, Ieronymaki E, Androulidaki A, Venihaki M, Margioris AN, Stathopoulos EN, Tsichlis PN, Tsatsanis C: Akt1 and Akt2 protein kinases differentially contribute to macrophage polarization. Proc Natl Acad Sci USA 2012;109:9517-9522.

$\checkmark 13$ Doxaki C, Kampranis SC, Eliopoulos AG, Spilianakis C, Tsatsanis C: Coordinated regulation of miR-155 and miR-146a genes during induction of endotoxin tolerance in macrophages. J Immunol 2015;15:5750-5761.

14 De Guire V, Robitaille R, Tétreault N, Guérin R, Ménard C, Bambace N, Sapieha P: Circulating miRNAs as sensitive and specific biomarkers for the diagnosis and monitoring of human diseases: promises and challenges. Clin Biochem 2013;46:846-860.

15 Andersson AM, Petersen JH, Jorgensen N, Jensen TK, Skakkebaek NE: Serum inhibin B and follicle-stimulating hormone levels as tools in the evaluation of infertile men: significance of adequate reference values from proven fertile men. J Clin Endocrinol Metab 2004;89:2873-2879.

16 Bobjer J, Katrinaki M, Tsatsanis C, Lundberg Giwercman Y, Giwercman A: Negative association between testosterone concentration and inflammatory markers in young men: a nested cross-sectional study. PLoS One 2013;8:61466.

17 Tsatsanis C, Bobjer J, Rastkhani H, Dermitzaki E, Katrinaki M, Margioris AN, Giwercman YL, Giwercman A: Serum miR-155 as a potential biomarker of male fertility. Hum Reprod 2015;30:853-860.

18 Murphy LE, Mills JL, Molloy AM, Qian C, Carter TC, Strevens H, Wide-Svensson D, Giwercman A, Levine RJ: Folate and vitamin B12 in idiopathic male infertility. Asian J Androl 2011;13:856-861.

19 Norlund L, Fex G, Lanke J, Von Schenck H, Nilsson JE, Leksell H, Grubb A: Reference intervals for the glomerular filtration rate and cell proliferation markers: serum cystatin $\mathrm{C}$ and serum beta 2 microglobulin/cystatin $\mathrm{C}$ ratio. Scand J Clin Lab Invest 1997;57:463-470. 
20 Brochner-Mortensen J: A simple method for the determination of glomerular filtration rate. Scand J Clin Lab Invest 1972;30:271-274.

-21 Krutzen E, Back SE, Nilsson-Ehle I \& Nilsson-Ehle P: Plasmaclearance of a new contrast agent, iohexol: a method for the assessment of glomerular filtration rate. J Lab Clin Med 1984;104:955-961.

22 World Health Organization: WHO Laboratory Manual for the Examination of Human Semen and SpermCervical Mucus Interaction (ed 4). Cambridge, Cambridge University Press, 1999.

23 Cooper TG, Björndahl L, Vreeburg J, Nieschlag E: Semen analysis and external quality control schemes for semen analysis need global standardization. Int J Androl 2002;25:306-311.

-24 Evenson D, Jost L: Sperm chromatin structure assay is useful for fertility assessment. Methods Cell Sci 2000;22: 169-189.

25 Erenpreiss J, Jepson K, Giwercman A, Tsarev I, Erenpreisa J, Spano M: Toluidine blue cytometry test for sperm DNA conformation: comparison with the flow cytometric sperm chromatin structure and TUNEL assays. Hum Repro 2004;19:2277-2282.

26 Iommiello V, Albani E, Di Rosa A, Marras A, Menduni F,Morreali G, Levi S, Pisano B, Levi-Setti P: Ejaculate oxidative stress is related with sperm DNA fragmentation and round cells. Int J Endocrinol 2015;321901.

-27 Izquierdo MJ, Cavia M, Muñiz P, de Francisco AL, Arias M, Santos J, Abaigar P: Paricalcitol reduces oxidative stress and inflammation in hemodialysis patients BMC Nephrol 2012;13:159.

28 Gunal, SY, Ustundag, B, Gunal, AI: The assessment of oxidative stress on patients with chronic renal failure at different stages and on dialysis patients receiving different hypertensive treatment. Indian J Clin Biochem 2013;28:390-395.

29 Akbari F, Alavi M, Esteghamati A, Mehrsai A, Djaladat H, Zohrevand R, Pourmand G: Effect of renal transplantation on sperm quality and sexhormone levels. BJU Int 2003;92:281-283.

30 De Celis R, Pedrón-Nuevo N: Male fertility of kidney transplant patients with one to ten years of evolution using a conventional immunosuppressive regimen. Arch Androl 1999;42:9-20. 